\title{
IMPROVEMENTS IN ENDF/B-VI IRON AND POSSIBLE \\ IMPACTS ON PRESSURE VESSEL SURVEILLANCE DOSIMETRY
}

\author{
C. I. Fu. D. M. Hetrick. C. M. Perer. F. G. Perey, N. M. Larson. D. C. Larson \\ Oak Ridge National Laboratory \\ Oak Ridge, TN 37831
}

CONF-900814--10

\section{ABSTRACT}

DE9 0016528

The ENDF/B-VI cross-section evaluations for the four iron isotopes are summarized, emphasizing the major improrements over ENDF/B-V. The evaluations were mostly based on a prelininary file generated in 1986 for natural iron that has been used for re-calculating several neutron-transport experiments, all of which showed improved agreement. These re-analyses, including those for pressure-vessel surveillance dosimetry, are also discussed.

\section{INTRODUCTION}

The results (Ref. 1 and references therein) from combining integral, differentiai, and calculated dosimetry data in the pressure vessels of several existing power reactors, using ENDF/B-V nuclear data, indicated that the iron inelastic scattering cross section in ENDF/B-V is about $8 \%$ too large from 3 tc $8 \mathrm{MeV}$. This indication was confirmed by newly available $\left(n, n^{\prime} \gamma\right)$ data ${ }^{2}$ and a new model calculation for ${ }^{56} \mathrm{Fe}$ using an improved nuclear model code ${ }^{3}$ to fit all of the available $\left(n, n^{\prime}\right)$ data and other relevant cross sections. All neutron-producing reaction cross sectirns above $3 \mathrm{MeV}$ in the ENDF/B-V iron evaluation were revised using the new model fits, resulting in a preliminary file ${ }^{4}$ for ENDF/B-VI. In this preliminary file, the energy-angle correlations for the inelastically scattered neutrons in the continuum were also introduced. The processed cross-section set ${ }^{5}$ in the Vitamin- $\mathrm{E}$ group structure ${ }^{6}$ has been collapsed to various broad-group structures for the re-analy'ses of several neutron-transport experiments, ${ }^{7-11}$ including the surveillance dosimetry mentioned above, all with improved agreement. The re-analysis ${ }^{7}$ of the neutron leakage spectrum of a thick iron-sphere experiment ${ }^{12}$ with a central $14-\mathrm{MeV}$ source showed that about half of the $100 \%$ increase in the leakage spectrum between 3 and $10 \mathrm{MeV}$ is due to the lowered inelastic scattering cross section in the new evaluation and the other half is due to the forward-peaked angular distributions introduced for the inelastically scattered neutrons in the continuum.

The test results from using the preliminary ENDF/B-VI evaluation for iron are summarized first. Other major improvements to be discussed are: (1) the separation of the evaluation into four isotopic files, (2) the use of the new File- 6 format for better representation of correlated energy-angle distributions of the outgoing neutrons and for introducing the energy distributions of the charged-particle and recoil spectra, and (3) the extension of the resonance parameter region of each isotope to higher neutron energy and the application of measured angular distributions for elastically scattered neutrons to determine the spins and parities of the resonances.

\section{TEST OF THE PRELIMINARY EVALUATION}

The preliminary ENDF/B-VI file for iron was intended to remore several outstanding discrepancies between integral data and calculations which occurred with the ENDF/B-V iron evaluation.

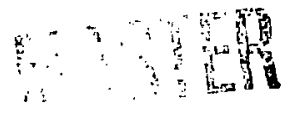


For a $14-\mathrm{Me} \mathrm{T}^{-}$source. the $\left(\mathrm{n} . \mathrm{n}^{\prime}\right)$ cross section for the continum (threshold $=4.5 \mathrm{Me} \mathrm{T}^{\prime}$ ! is important for any neutron transport problem. It is well known ${ }^{3}$ that inelastically. scattered neutrons in the continum are forward-peaked. Forward-peaked neutrons are more penetrating, hence the isotropic assumption for the inelastically scattered neutrons in the continum in ENDF/B-Y iron is no doubt a source of error for thich-shield analysis involving fusion sources. 12

In the cases of fission problems. analyses of three integral measurements consistently indicated that the $\mathrm{Fe}\left(n, n^{\prime}\right)$ cross section in ENDF/B-V above $3 \mathrm{MeV}$ was too large. The three problens are: (1) the pressure ressel surveillance dosimetry for several existing power reactors, ${ }^{1.8}(2)$ the source terms for the Hiroshima and Tagasaki dosimetry reevaluation program, ${ }^{10}$ and $(3)$ the Tower Shielding Facility deep penetration measurements. ${ }^{11}$

New Fe( $\left.n, n^{\prime} \gamma\right)$ data for the $846-\mathrm{keV}$ level ${ }^{2}$ and the new TXG code ${ }^{3}$ capable of calculating angular distributions for continum neutrons were used for addressing the above two types (fission and fusion) of problems and generating the preliminary file. The corresponding multigroup cross-section set ${ }^{6}$ was applied to the four experiments mentioned above, all showing improved agreement with the data. ${ }^{7-11}$ Smaller discrepancies remain, but they are rather difficult to resolve. For example, Maerker ${ }^{8,9}$ suggested that the $\operatorname{Fe}\left(n, n^{\prime}\right)$ cross section below $3 \mathrm{MeV}$ is still a few percent too large but we don't have any new experjmental data or theoretical means (because the cross section below $3 \mathrm{MeV}$ is structured) to answer this question at present. Additional high-resolution ( $5 \mathrm{heV}$ ) and/or high-accuracy (2\%) measurement in this energy region are needed.

The ENDF/B-VI iron evaluation retains the basic features of the prelimirary file. Figure 1 compares the $\mathrm{Fe}\left(n, n^{\prime}\right)$ cross sections between ENDF/B-VI and -V. The newly available data of Larson $^{2}$ are also shown. The ENDF/B-VI results shown are abundanceaverages of the isotopic evaluations and closely resemble those shown for the preliminary file, ${ }^{4}$ where older data were also plotted.

Figure 2 illustrates the calculated and experimental ${ }^{13-15}$ double differential $\mathrm{Fe}(n, x n)$ cross sections for $14.5-\mathrm{MeV}$ incident neutrons and several secondary energy ranges. Equally good agreement between calculation and experiment has been shown ${ }^{4}$ for the incident energy of $26 \mathrm{MeV}$, suggesting reliability of the model used for the calculation. The ENDF/B$\mathrm{V}$ data, if plotted in Fig. 2, would appear as horizontal lines.

\section{INTRODUCTION OF ISOTOPIC EVALUATIONS}

The isotopic evaluations enabled us to make use of additional data measured for the individual isotopes. For example, careful analyses of the inelastic scattering cross sections of the minor isotopes make the values of the inelastic cross section for natural iron between 1.4 and $3.4 \mathrm{MeV}$ about $2 \%$ smaller, a change that would not have drawn our attention without evaluating the cross sections of the minor isotopes separately. This reduction in the inelastic scattering cross section of natural iron may result in improved agreement between calculated and measured ${ }^{237} \mathrm{~Np}(n, f)$ responses in the pressure vessels of existing power reactors. However, confirmation of it will await processing of the new evaluation and re-analysis of the surveillance dosimetry data.

The separation of the natural iron evaluation into four isotopic evaluations allows the representation of activation and dosimetry reaction cross sections in the general purpose files. For example, the dosimetry reactions ${ }^{54} \mathrm{Fe}(n, p)$ and ${ }^{56} \mathrm{Fe}(n, p)$ are now contained in the respective general purpose files. These two cross sections were ufdated for ENDF/BVI primarily by using the ENDF/B-VI ${ }^{235} \mathrm{U}(n, f)$ and ${ }^{238} \mathrm{U}(n, f)$ standards in the GLLCS package containing 14 correlated reactions. ${ }^{16}$ The evaluation of 37 high-priority dosimetry and activation cross sections available in the general purpose files for which we are responsible has been summarized. ${ }^{17}$ 
In an evaluated cross-section file for an element containing several iscoropes. it is ust possible to represent the energies of the reaction products correctly. hence the energy balance cannot be guaranteed. For example. the Q-values for the (n. - ) erents for ${ }^{5} \mathrm{~F} e$ and ${ }^{5} \mathrm{Fe}$ are respectively: 9.3 and $7.8 \mathrm{Mel}$. This difference in the capture gamma-ray ex ergies can be correctly accounted for only in the new isotopic evaluations. ensuring energy balance and more reliable estimates of derived quantities such as lierma factors. In the area of radiation damage studies. the new File- $\theta$ format plays an important role. which is explained below.

\section{USE OF FILE-6 FORMAT}

Correlated energy-angle distributions of outgoing neutrons can now be given efficiently in the new File-6 format in ENDF/B-VI. In the case of the iron isotopes, correlated energyangle distributions are given for the out going neutrons for each neutron-producing reaction, and energy distributions are available for the emitted protons, alpha-particles, heary recoils. and gamma rays. The incident neutron energy and the sum of the average energies of all reaction products have been checked to be consistent within $1 \%$. The fact that energies are balanced and the fact that energy distributions of heary recoils are given should have a positive impact on derived quantities such as displacement cross sections for radiation damage studies.

\section{RESONANCE PARAMETERS}

SAMMY, ${ }^{18}$ an R-matrix code based on the Reich-Moore formalism and Bayes' Equations, was used for the resonance region of the iron isotopes. For ${ }^{56} \mathrm{Fe}$, the analysis was based on measured cross sections for total ${ }^{19}$ and capture, ${ }^{20}$ and the angular distributions for neutron elastic scattering. ${ }^{19}$ The resolved resonance region for all isotopes were extended to higher energy than available in ENDF/B-V. In the case of ${ }^{56} \mathrm{Fe}$, the resonance region was increased from 400 to $850 \mathrm{keV}$. An example is shown in Fig. 3 where the measured transmission data ${ }^{19}$ and elastic angular distributions ${ }^{19}$ jointly determine the spin and parity assignments of several resonances. The new ENDF/B-VI formats allow the calculation of elastic angular distributions in the resonance region directly from the resonance parameters. It has been shown ${ }^{19}$ that resonance parameters evaluated without considering the scattering data often resulted in incorrect spin and parity assignments and hence poor results for the calculated angular distributions. The ENDF/B-V data in the energy region shown in Fig. 3 agree well with the ENDF/B-VI results shown; therefore they are omitted. Howerer, angular distributions for the elastically scattered neutrons, such as shown in the three lower graphs of Fig. 3, cannot be obtained from ENDF/B-V.

\section{CONCLUDING REMARKS}

Five major improrements in the ENDF/B-VI evaluation for iron orer Version $V$ have been summarized, namely, (1) the consideration of integral data in a preliminary ENDF/BVI evaluation, (2) the introduction of energy-angle correlations for the nonelastically scattered neutrons in the continuum, (3) the separation of the evaluation into four isotopic files, (4) the use of the new File- 6 format for charged-particle energy distributions and energy balance assurance, and (5) the extension of the resolved resonance parameters to higher energies and the ability to calculate angular distributions for the elastically scattered neutrons in the resonance encrgy range. The first two have been positively tested, 
particularly in the surveillance dosimetry area. The other three improvements are solidly based on adranced model analyses of a rather large data base.

\section{ACKNOWLEDGMENTS}

The authors wish to thank Drs. J. K. Dickens and R. E. Maerker for helpful comments and discussions. This research was sponsored by the Office of Energy Research, U. S. Department of Energy, under contract DE-AC05-840R21400 with Martin Marietta Energy Systems, Inc.

\section{REFERENCES}

1. R. E. Maerker et al., "Combining Integral and Differential Dosimetry Data in an Unfolding Procedure with Application to the Arkansas Nuclear One-Unit 1 Reactor," p. 477, Proc. Int. Conf. Nucl. Data for Basic and Applied Sciences, Santa Fe, May 13-17, 1985.

2. D. C. Larson, "High-Resolution Structural Material $(n, x \gamma)$ Production Cross Sections for $E_{n}$ from 0.2 to $40 \mathrm{MeV}$," p. 71 , Proc. Int. Conf. Nucl. Data for Basic and Applied Sciences, Santa Fe, May 13-17, 1985.

3. C. Y. Fu, Nucl. Sci. Eng. 100,61 (i08s).

4. C. Y. Fu and D. M. Hetrick, Update of ENDF/B-V Mod-3 Iron: Neutron-Producing Reaction Cross Sections and Energy-Angle Correlations, ORNL/TM-9964 (ENDF341), 1986.

5. R. W. Roussin and J. I. Bartley, Oak Ridge National Laboratory, private communication (1986).

6. C. R. Weisbin et al., Vitamin-E: An ENDF/B-V Multigroup Cross-Section Library for LMFBR Core and Shield, LWR Shield, Dosimetry and Fusion Blanket Technology, ORNL-5505 (ENDF-274), Oak Ridge National Laboratory (1979).

7. C. Y. Fu and D. M. Hetrick, "Evaluation and Testing of Double Differential Fe $\left(n, n^{\prime}\right)$ Cross Sections," Trans. Am. Nucl. Soc. 53, 409 (1986).

8. R. E. Maerker, Nucl. Sci. Eng. 96, 288 (1987).

9. R. E. Maerker, Analysis of the NESDIP2 and NESDIP3 Radial Shield and Cavity Experiments, ORNL/TM-10389, Oak Ridge National Laboratory (1987).

10. J. V. Pace, Oak Ridge National Laboratory, private communication (1986).

11. C. O. Slater, JASPER Workshop, Oak Ridge National Laboratory, April 1987.

12. R. H. Johnson, J. J. Dorning, and B. W. Wehring, Trans. Am. Nucl. Soc. 22, 799 (1975).

13. J. L. Kammerdiener, Neutron Spectra Emitted ty Fe Irradiated by $14 \mathrm{MeV}$ Neutrons, UCRL-51232, Lawrence Livermore Laboratory, 1972.

14. D. Hermsdorf et al., Differential Neutron Emission Cross Sections from Iron by 14.6 MeV Neutrons, ZfK-277, Institute of Nuclear Physics, University of Dresden, 1975.

15. A. Takahashi et al., "Double Differential Neutron Emission Cross Sections, Numerical Tables and Figures," A-83-01, Intense 14-MeV Neutron Source Facility, Osaka University, 1983.

16. C. Y. Fu and D. M. Hetrick, "Experience in Using the Covariances of Some ENDF/BV Dosimetry Cross Sections: Proposed Improvements and Addition of Cross-Reaction Covariances", p. 877, Proc. 4th ASTM-EURATOM Symposium on Reactor Dosimetry, NUREG/CP-0029, Gaithersburg, Maryland, March 22-26, 1982.

17. C. Y. Fu, "A Review of Activation Cross Sections in the ENDF/B-VI General Purpose Files for $\mathrm{Cr}, \mathrm{Fe}, \mathrm{Ni}, \mathrm{Cu}$, and $\mathrm{Pb}, "$ p. 275 , Proc. NEANDC Specialists' Meeting on Neutron Activation Cross Sections for Fission and Fusion Energy Applications, NEANDC-259 'U', Argonne National Laboratory, Sept. 13-15, 1989. 
1S. N. M. Larson and F. G. Perey; Lsers' Guide for SAMMM: A Computer iivdel for ifulti-Level R-Matrix Fits to Neutron Data L'sing Bayes' Equations, ORNL/TM-7485 (1980); Lpdates ORNL/TM-9179 (1984), ORNL/TM-9179/R1 (1985) and /R2 (19S9).

19. C. M. Perey et al., Oak Ridge National Laboratory, to be published (1990).

20. F. Corvi et al., Proc. Consultants Meeting on Miclear Data for Structural Materials. Vienna, Austria, 1983, International Nuclear Data Committee Report INDC(NDS)-152 L, 1984 and private communication to C. M. Perey, 1984. 


\section{FIGURE CAPTIONS}

Fig. 1. Comparison of the Fe inelastic cross sections of ENDF/B-VI with ENDF/B-Y and with experimental data of Larson. ${ }^{2}$

Fig. 2. Calculated and experimental ${ }^{13.14,15}$ double differential $F e(n, x n)$ cross sections for $14.5-. \mathrm{MeV}$ incident neutrons and several secondary energy ranges.

Fig. 3. In the upper graph the theoretical total cross sections, calculated with the parameters of ENDF/B-VI ${ }^{56} \mathrm{Fe}$, are compared with the data. ${ }^{19}$ Parentheses are used to indicate uncertain spin and parity assignments. The assignments were made using the differential elastic scattering data ${ }^{\text {ig }}$ shown in the three lower graphs. 
nat-Fe TOTAL INELASTIC SCATTERING CROSS SECTION

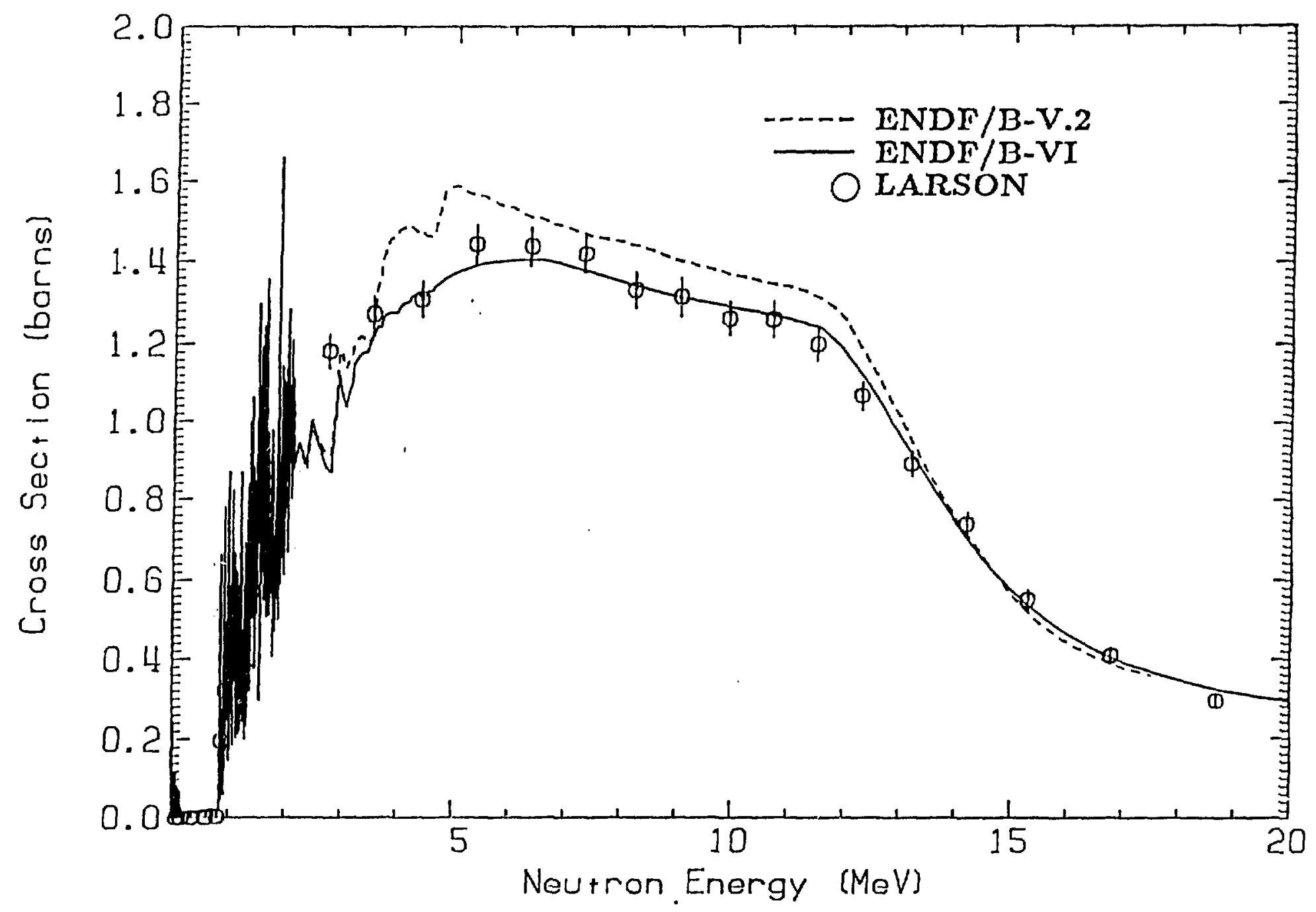




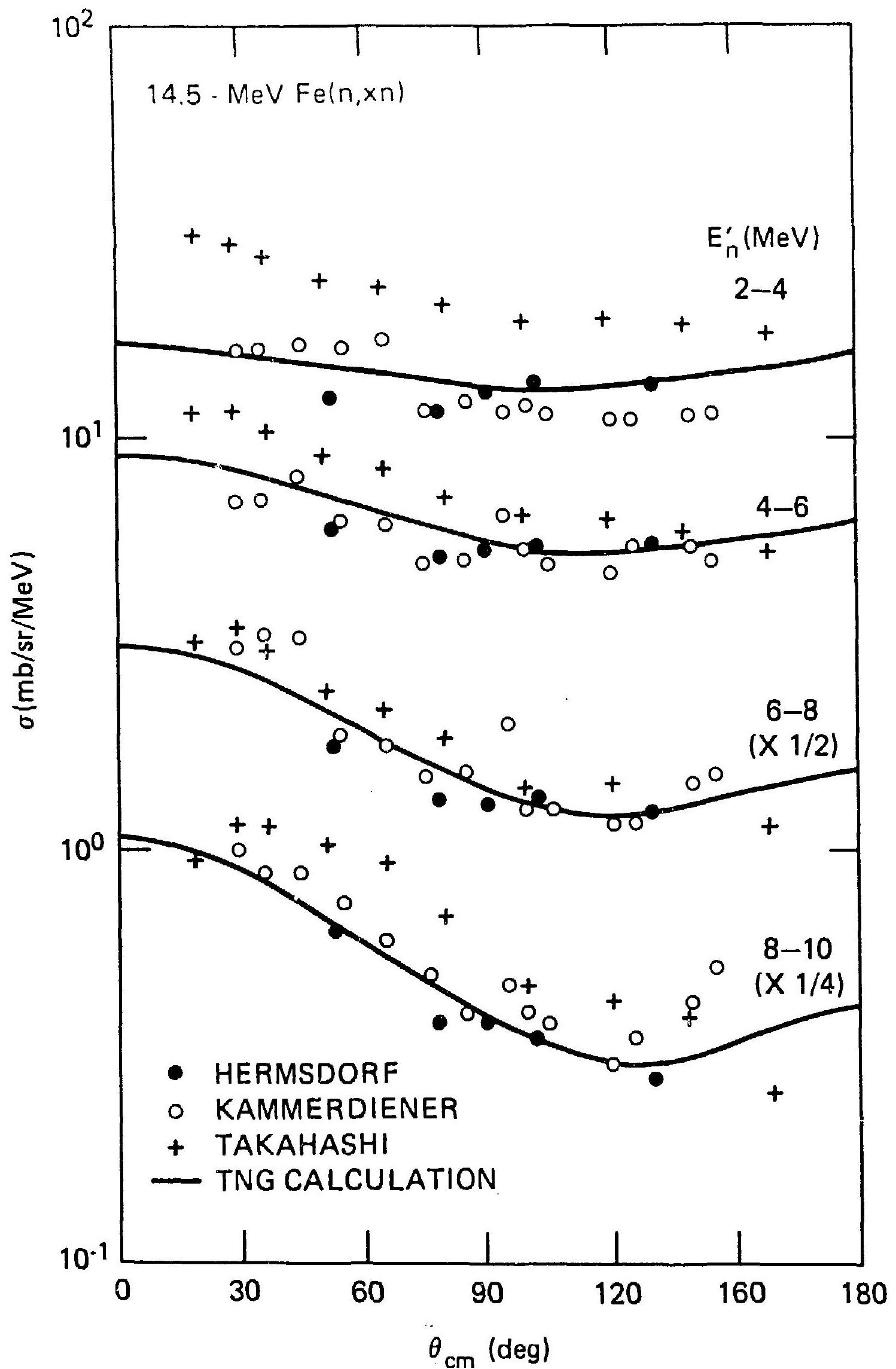




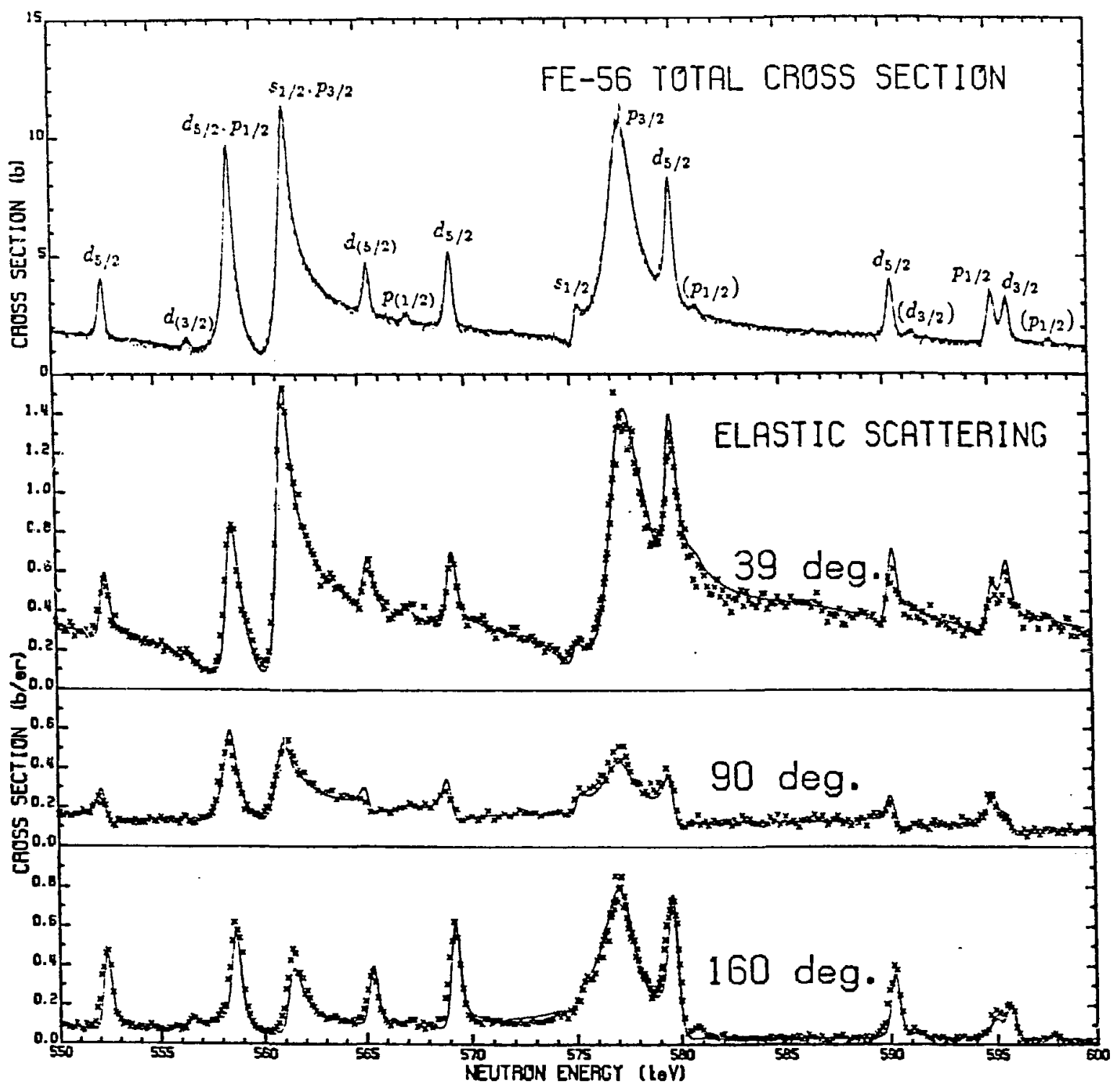

